(Aus der Giessener Universitäts-Frauenklinik.)

\title{
Die Schätzung der Transversa des Beckeneingangs nach Löhlein, nachgepriift an 74 Bänderbecken.
}

Von

\section{Dr. Martin Steinbrecher,}

npprob. Arzt ans Kreuznach.

(Mit 2 Figuren im Text.)

Unter den Ursachen regelwidriger Geburten nimmt das enge Becken zweifellos die erste Stelle ein, es beherrscht, wie Spiegelberg $\left.{ }^{1}\right)$ sich ausgedrückt hat, geradezu die Pathologie der Geburt. Und so sehen wir, dass seit Deventer ${ }^{2}$ ), der zuerst uns auf das enge Becken aufmerksam machte, fast alle bedeutenden Geburtshelfer sich um die Lösung dieses wichtigen Problemes bemühten und verdient machten.

In erster Linie kam es darauf an, das enge Becken als solches zu erkennen, und dies konnte nur geschehen durch exacte Messungen. Baudeloque ${ }^{3}$ ) war der erste, der auf diesem Gebiete bahnbrechend vorging. Während er den Grund zur äusseren Beckenmessung legte, lässt sich die innere Beckenmessung auf Smellie ${ }^{4}$ ) zurückführen.

So sehr es auch im Laufe der Zeit gelang, die äussere Beckenmessung zu vervollkommnen und auszubauen, so waren ihre Resultate doch nicht im Stande, uns über die Raumverhältnisse im kleinen Becken Aufschluss zu geben, wenigstens nicht, darin werden alle Geburtshelfer einig sein, in pathologischen Fällen.

1) Spiegelberg, Lehrb. d. Geburtsh. 1882. 2. Aufl. S. 392.

2) Henricus a Deventer, Novum lumen obstetr. Lugd. Bat. 1701. Deutsch: Neues Hebammenlicht. Jena 1717. (6. Aufl. 1755.) Chap. 27.

3) Baudeloque, L'art des acoouchements 1781.

4) Smellie, A collection of cases. London 1754 . 
Man musste also den von Smellie gewiesenen Weg betreten and die innere Beckenmessung als nothwendiges diagnostisches Hülfsmittel zu Rathe ziehen. Auch diese Art der Messung suchte man zu vervollkommnen: neben der manuellen begründete man eine instrumentelle innere Messung ${ }^{1}$ ). Was man auf diesem Wege erreicht hat, darüber belehrt uns am Besten ein Einblick in die Lehrbücher der Jetztzeit:

So sagt $B u m^{2}$ ): "Es ist schon viel Scharfsinn darauf verwendet worden, Instrumente zu construiren, welche eine genaue Abmessung der Beckenhöhle von innen her gestatten sollen. In der Praxis hat sich bis jetzt noch kejner der zahlreichen Beckenmesser einbürgern können. Dazu ist ihre Handhabung zu complicirt und zu schmerzhaft. Der beste Beckenmesser ist deshalb immer noch die Hand, mittelst deren wir den wichtigsten Durchmesser des Eingangs, die Conj. vera, auf einfache und zuverlässige Art bestimmen können."

Bezüglich des Querdurchmessers sagt er dann weiter: "Für die exacte Messung des Querdurchmessers des Beckeneingangs lässt die Hand im Stich. Nan begnügt sich gewöhnlich mittelst zweier Finger oder der halben Hand die seitlichen Wände des Beckenraumes abzutasten und sich so schätzungsweise ein Urtheil über seine etwaige Verengerung in der Querrichtung zu verschaffen; sind die Resultate auch nicht genau, so reicht man damit in der Praxis doch aus."

In v. Winckel's Handbuch der Geburtshülfe ${ }^{3}$ ) widmet E. Sonntag der instrumentellen inneren Beckenmessung einen besonderen Abschnitt. Nach einigen kritisch-historischen Auslassungen über die diesbezüglichen bemerkenswerthen Instrumente fährt er fort: "Von all' den vielen Methoden der instrumentellen Beckenmessung hat bisher noch keine einzige eine allgemeine Verbreitung erlangt - , ein Beweis dafür, dass ein allen Anforderungen entsprechendes Verfahren oder Messinstrument noch nicht gefunden worden ist. Für das Bedürfniss der Praxis genügt unstreitig, sofern sie in der geschilderten Weise sorgfältig ausgeführt wird, die innere Abtastung und digitale Messung des Beckens" u.s. w.

Ferner: „Wenn die Feststellung der inneren Messpunkte der

1) Begründet von Stein d. Aelteren.

2) Bumm, Grundriss d. Geburtsh. 1903. S. 334.

3) v. Wincke1, Handb. d. Geburtsh. Bd. II. Theil 3. 
Schätzung einen weiten Spielraum gewährt, so gilt das insbesondere von der instrumentellen Messung des Querdurchmessers im Beckeneingang, die noch immer ein nur unvollkommen gelöstes Hauptproblem der ganzen Beckenuntersuchung bildet."

Olshausen ${ }^{1}$ ) erwähnt in seinem Lehrbuche die innere Beckenmessung nur in aller Kürze, indem er auf die Methoden von Küstner, Kehrer und Löhlein hinweist. Bezüglich des queren Durchmessers des Beckeneingangs sagt er: "es fehlt uns eine Methode, durch die wir eine halbwegs genaue Zahl für die Grösse dieses Durchmessers erhalten können, noch vollkommen."

Ahlfeld ${ }^{2}$ ) bemerkt in seinem Lehrbuche der Geburtshülfe auf S. 351: "Für die gewöbnliche Praxis genügt die Hand und das Centimetermaass zur Ausführung der Beckenmessung. Die Unzahl von Maassinstrumenten, die im Laufe der Jahre angegeben und wieder obsolet geworden sind, beweist, dass wir noch kein Instrument besitzen, dass der Hand vorzuziehen sei. Werden Beckenmessungen zu wissenschaftlichen Zwecken ausgeführt, so benutze man die von Skutsch angegebenen Instrumente und Methoden."

In seiner kurz gefassten Geburtshülfe erwähnt Fritsch ${ }^{3}$ ) die instrumentelle, innere Beckenmessung überhaupt nicht, ebenso geht er nicht auf die Bestimmung der Transversa des Beckeneingangs ein.

Kaltenbach ${ }^{4}$ ) macht folgende Bemerkungen: "Für die Messung des Querdurchmessers des Beckeneingangs haben Freund und Küstner recht sinnreiche Methoden angegeben, doch haben auch diese mehr wissenschaftlich-didactischen als praktischen Werth." Es folgt dann eine kurze Beschreibung der Freund'schen Methode.

Optimistischer klingen die Auslassungen Schauta's in seiner operativen Geburtshülfe ${ }^{5}$ ); er erwähnt für die innere instrumentelle Messung nur die Methode von Skutsch und glaubt, dass damit die. innere instrumentelle Beckenmessung $n^{\mathrm{zu}}$ einem befriedigenden Abschluss gekommen" ist.

Aehnlich sagt Zweifel in seinem Lehrbuche der Geburtshülfe ${ }^{6}$ ) S. 161: "Nach vielfachen Erfahrungen kann ich diese

1) Olshausen u. Veit, Geburtshülfe. 13. Aufl. S. 620.

2) Ahlfeld, Lehrb. d. Geburtsh. 1903. III. Aufl.

3) Fritsch, Geburtshülfe 1904.

4) Kaltenbach, Lehrb. d. Geburtsh. 1893.

5) Schauta, Operative Geburtshülfe 1892. II. Aufl.

6) Zweifel, Lehrb. d. Geburtsh. 1903. 5. Aufl. 
(von Skutsch angegebene) Messung der Conj. vera, überhaupt aller inneren Beckenmesser warm empfehlen."

Aus dieser Sammlung von Ansichten und Urtheilen geht in erster Linie die Stellung der Autoren zur inneren instrumentellen Beckenmessung überhaupt hervor. Die Mehrzahl verhält sich ihr gegenüber ablehnend, einige heben ihren didaktischen Werth hervor, und nur wenige sprechen sich für ihre Anwendung aus. Diese Stellungnahme scheint unseres Erachtens wesentlich ron dem Grad der Wichtigkeit beeinflusst, den die einzelnen Autoren der Bestimmung der Transversa des Beckeneingangs beimessen.

Mehr als die genannten Autoren tritt Skutsch für eine exacte innere Messung ein. In seinem bekannten Buche ${ }^{1}$ ), in welchem er uns neben einer genauen Geschichte der Beckenmessung auch den schon mehrfach erwähnten, von ihm erfundenen Beckenmesser schenkt, sagt er: "Alle Durchmesser des Beckens müssen in gleicher Weise unserer Diagnose zugeführt werden. Die genaue Kenntniss der Verkürzung der queren Durchmesser ist in ebensolchem Maasse für die richtige Indicationsstellung maassgebend, wie die Verkürzung der geraden Durchmesser. Nur eine sichere Kenntniss der Weite bezw. Engigkeit des gesammten Beckencanals darf unsere Ansprüche auf Exaktheit befriedigen."

Neben Skutsch war vor Allem auch Küstner eifrig bemüht, der Lösung des Problems der inneren Beckenmessung näher zu kommen. Von seinen 3 Methoden hat schliesslich nur die letzte ${ }^{2}$ ) eine grössere Verbreitung gefunden; es ist dies eine directe, instrumentelle Methode zur Messung des Querdurchmessers.

In ähnlicher Weise war auch Löhlein ${ }^{3}$ ) von der Wichtigkeit der Transversa überzengt, er spricht von ihr als dem "Durchmesser, der an Wichtigkeit gleich hinter der geburtshülflichen Conjugata kommt, dessen Bestimmung immer die grössten Schwierigkeiten geboten hat, und dessen Nichtberücksichtigung am häufigsten folgenschwere Missgriffe bedingt".

Diese Ueberzeugung veranlasste anch Löhlein, auf diesem Gebiete Forschungen anzustellen, nur verliess er den Weg der instrumentellen Methode. Er bemühte sich längere Zeit für die Schätzung der Transversa Maasse und Beziehungen zu finden, die S. 94.

1) Skutsch, Die Beckenmessung an der lebenden Frau. Jena 1887.

2) Küstner, Verhandl. auf dem I. Congress für Gyn. München 1886.

3) Löhlein, Zeitschr. f. Gyn. u. Geb. Bd. XI. 
es ermöglichen sollten, durch manuelle Messung indirect zum Ziele zu gelangen. Endlich fand er folgende für das normale Becken ziemlich constante Beziehung: Er verband die Mitte des Lig. areuat. der Symphyse mit dem vorderen oberen Winkel der Incis. ischiad. maj. und erhielt so eine Linie, die er die Schrägaufsteigende nannte. Diese Linie soll nun die Eigenschaft haben, dass sie um eine Constante vermehrt, die Transversa des Beckeneingangs ergiebt, oder genauer, wenn man die halbe Summe beider Schrägaufsteigenden um eine gewisse Zahl vermehrt, soll man den Querdurchmesser des Eingangs erhalten. (Also, wenn man die Schrägaufsteigenden mit $l_{1}$ und $l_{2}$, die Constante mit $c$ bezeichnet, so würde die Formel lauten: $\frac{l_{1}+l_{2}}{2}+c=$ Transversa.) Diese Methode der digitalen Messung ist nach Löhlein mit einiger Uebung auch an der Lebenden ausführbar.

Stimmten diese Angaben in dem genannten Umfange, d. h. ohne jede Einschränkung, so müsste man sagen, dass wir damit in der inneren Beckenmessung einen grossen Schritt vorwärts gebracht worden wären, wir hätten eine einfache Methode, ähnlich der für die Bestimmung der Conj. vera, wir hätten wie dort den Vortheil, ohne Instrumente auskommen zu können, dem Praktiker vor Allem wäre geholfen, bei dem sich auch das Skutsch'sche Instrument noch nicht hat einbürgern können. Doch leidèr ist dem nicht so. Sehon in der Publicationsschrift giebt Löhlein selbst die beschränkte Anwendbarkeit der Vethode zu und er schliesst mit den Worten: "Meine Bemühungen würden reichlich belohnt sein, wenn wir auch nur für das gleichmässig allgemein verengte Becken und diesen nahestehenden Formen des allgemein verengten platten Beckens zur Schätzung der Transversa in der Messung des Abstandes zwischen Lig. arcuat. und Incis. ischiad. einen bestimmten Anhalt und für die Austastung der Beckenhöhle eine gewisse Basis gewonnen hätten."

Als wichtigstes Ergebniss seiner Arbeit, welcher Messungen an 21 Bänderbecken zu Grunde liegen, hebt Löhlein in gesperrtem Druck hervor: "Demnach ist man meines Erachtens berechtigt, für die Schätzung der Querdurchmesser nicht deformer Becken die Distanz vom Lig. arcuat. bis zum vorderen oberen Winkel des Foramen ischiad. maj. in der Art zu verwerthen, dass man zu ihr im Mittel $20 \mathrm{~mm}$ addirt, um die Transversa annähernd zu erhalten. Die zu addirende Zahl wird dann etwas niedriger anzunehmen sein $(20-15 \mathrm{~mm})$, wenn die Symphyse hoch und wenig geneigt und die Verkürzung der geraden Durchmesser eines durchweg zu engen Beckens hinter derjenigen der queren zurïekzusteben scheint." 
Man bätte nun nach der Publication dieser neven Methode annehmen sollen, dass gerade ihre Einfachheit viele hätte veranlassen müssen, die Methode nachzuprüfen. Dies scheint aber nicht der Fall gewesen zu sein, wie sich schon aus dem Mangel an diesbezüglicher Literatur ergiebt. In den Lehrbüchern wird sie fast gar nicht mehr erwähnt, Olshausen nennt sie nur dem Namen nach und Sonntag, der sie erwähnt ${ }^{1}$ ), fällt ein abfälliges Urtheil über sie, indem er sagt, die Methode passe nur für normale Becken und könne deshalb nur eine untergeordnete Bedeutung beanspruchen. Die Methode ist dermassen in Vergessenheit gerathen, dass auch Geburtshelfer von Fach sie kaum noch dem Namen nach kenner. Nur in der Giessener Klinik, soweit uns bekannt, ist man seit Löhlein der Methode treu geblieben, und man möchte sie daselbst auch nicht missen, weil man die Ueberzeugung hegt, dass sie wesentlich dazu beiträgt, uns über die räumlichen Verhältnisse des kleinen Beckens eine einigermaassen befriedigende Vorstellung zu geben.

Diese Ueberzeugung veranlasste auch Herrn Geh. Rath Prof. Pfannenstiel: den Verfasser eine Nachprüfung der Löhlein'schen Methode an den in der Klinik vorhandenen Bänderbecken vornehmen zu lassen, deren Resultate im Folgenden mitgetheilt werden sollen.

Gemessen wurden 74 Becken, von denen weitaus die meisten zu den pathologischen Formen gerechnet werden müssen. Die Messungen, die an sich leicht sind, wurden sämmtlich mit dem Finger ausgeführt - dabei musste natürlich die rechte Seite mit der rechten Hand, die linke mit der linken ausgetastet werden. Die Controlle der digitalen durch die Stabmessung ergab, dass die digitale Messung mit hinreichender Sicherheit arbeitete. In die Tabellen wurden folgende Maasse aufgenommen: Conj. vera, Conj. diagon., der quere und die beiden schrägen Durchmesser des Beckeneingangs, die beiden Schrägaufsteigenden Löhlein's, der Durchschnitt dieser beiden letzteren und die Differenz zwischen dem Durchschnitt und der Transversa (also die Constante $C=\operatorname{Tr}-\frac{l_{1}+l_{2}}{2}$ ). Die verschiedenen Beckenformen sind allgemein geordnet; es wurden unterschieden: normale, übernormale, allgemeinverengte, platte, allgemgeinverengt-platte, schrägverschobene-platte, schrägverengte und querverengte Formen. Die Maasse sind der Gleichmässigkeit halber in Millimeter angegeben.

1) v. Winckel, Handbuch der Geburtshülfe. II. 3. S. 1856. 
Tabelle I. Normale Becken.

\begin{tabular}{|c|c|c|c|c|c|c|c|c|c|}
\hline No. & C. v. & Tr. & C. d. & Obl. d. & Obl. s. & $\begin{array}{l}\text { Lö } \\
\text { R. }\end{array}$ & $\begin{array}{l}\text { in } \\
\text { L. }\end{array}$ & $\begin{array}{l}\text { Dureh- } \\
\text { schnitt }\end{array}$ & Differ. \\
\hline $\begin{array}{l}1 \\
2 \\
3 \\
4 \\
5\end{array}$ & $\begin{array}{l}110 \\
110 \\
110 \\
110 \\
105\end{array}$ & $\begin{array}{l}136 \\
133 \\
130 \\
130 \\
133\end{array}$ & $\begin{array}{l}130 \\
131 \\
120 \\
129 \\
125\end{array}$ & $\begin{array}{l}\overline{133} \\
\overline{121} \\
128\end{array}$ & $\begin{array}{l}\overline{135} \\
\overline{121} \\
128\end{array}$ & $\begin{array}{l}120 \\
118 \\
111 \\
117 \\
120\end{array}$ & $\begin{array}{l}118 \\
120 \\
115 \\
117 \\
120\end{array}$ & $\begin{array}{l}119 \\
119 \\
113 \\
117 \\
120\end{array}$ & $\begin{array}{l}17 \\
14 \\
17 \\
13 \\
13\end{array}$ \\
\hline & & & & & & \multicolumn{2}{|c|}{ Summe: } & 588 & 74 \\
\hline & & & & & & \multicolumn{2}{|c|}{ Durchschnitt: } & 117,6 & 14,8 \\
\hline
\end{tabular}

Schlussfolgerung: Die Schrägaufsteigenden bewegen sich normaliter in Grenzen von 11 und $12 \mathrm{~cm}$ (absolute Maasse von 11,1-12 cm). Die zu addirende Differenz schwankt zwischen 1,3 und $1,7 \mathrm{~cm}$ and beträgt im Durchschnitt ca. $1,5 \mathrm{~cm}$.

Tabelle II. Uebernormale Becken.

\begin{tabular}{|c|c|c|c|c|c|c|c|c|c|}
\hline No. & C. v. & $\operatorname{Tr}$. & C. d. & o. d. & o. s. & L. R. & L. I. & $\begin{array}{l}\text { Dureh- } \\
\text { schnitt }\end{array}$ & Differ. \\
\hline 6 & 120 & 143 & 138 & - & - & 129 & 130 & 129,5 & 13.5 \\
\hline 7 & 110 & 140 & 130 & 125 & 137 & 122 & 126 & 124 & 16 \\
\hline 8 & 116 & 135 & 132 & - & - & 122 & 122 & 122 & 13 \\
\hline & & & & & & \multicolumn{2}{|c|}{ Summe: } & 375,5 & 42,5 \\
\hline & & & & & & \multicolumn{2}{|c|}{ Durchschnitt: } & 125,1 & 14.16 \\
\hline
\end{tabular}

Schlussfolgerung: Die übernormalen Becken verhalten sich im wesentlichen wie die normalen; die Durchschnittsdifferenz bleibt nur wenig hinter der der normalen zurück.

Tabelle III. Allgemeinverengte Becken.

\begin{tabular}{|c|c|c|c|c|c|c|c|c|c|}
\hline No. & C. v. & Tr. & C. d. & o. d. & o. s. & L. R. & L. L. & $\begin{array}{l}\text { Durch- } \\
\text { schnitt }\end{array}$ & Differ. \\
\hline 9 & 100 & 120 & 122 & - & - & 108 & 106 & 107 & 13 \\
\hline 10 & 100 & 130 & 110 & 120 & 120 & 102 & 105 & 103,5 & 26,5 \\
\hline 11 & 100 & 125 & 120 & 118 & 116 & 112 & 112 & 112 & 13 \\
\hline 12 & 105 & 125 & 123 & 120 & 128 & 109 & 115 & 112 & 13 \\
\hline 13 & 93 & 115 & 102 & - & - & 100 & 95 & 97,5 & 17,5 \\
\hline 14 & 101 & 125 & 124 & 120 & 125 & 115 & 120 & 117,5 & 7,5 \\
\hline 15 & 102 & 130 & 118 & 121 & 118 & 115 & 117 & 116 & 14 \\
\hline 16 & 100 & 125 & 116 & 118 & 116 & 112 & 113 & 112,5 & 12,5 \\
\hline 17 & 106 & 127 & 119 & 125 & 121 & 120 & 118 & 119 & 8 \\
\hline 18 & 105 & 125 & 118 & 118 & 118 & 112 & 112 & 112 & 13 \\
\hline 19 & 102 & 133 & 123 & 124 & 124 & 119 & 119 & 119 & 14 \\
\hline 20 & 106 & 130 & 125 & 125 & 127 & 125 & 115 & 120 & 10 \\
\hline & & & & & & \multicolumn{2}{|c|}{ Summe: } & 1348 & 162 \\
\hline & & & & & & \multicolumn{2}{|c|}{ Durchschnitt: } & 112,3 & 13,5 \\
\hline
\end{tabular}


Schlussfolgerung: Entsprechend der allgemeinen Verengung. sind auch die Löhlein'schen Masse durchschnittlich klein: 11,22 mm; die absoluten Maasse bewegen sich in Grenzen von 9,5 und $12,5 \mathrm{~cm}$. Auch die constante (Differenz) ist geringer, was ja $z u$ erwarten war, sie beträgt $13,5 \mathrm{~mm}$.

Tabelle IV. Platte Becken.

\begin{tabular}{|c|c|c|c|c|c|c|c|c|c|}
\hline No. & C. v. & Tr. & C. d. & $0 . d$. & o.s. & L. R. & L. L. & $\begin{array}{l}\text { Dureh- } \\
\text { schnitt }\end{array}$ & Differ. \\
\hline $\begin{array}{l}21 \\
22 \\
23 \\
24 \\
25 \\
26 \\
27 \\
28 \\
29 \\
30 \\
31 \\
32 \\
33 \\
34 \\
35 \\
36 \\
37 \\
38 \\
39 \\
40 \\
41\end{array}$ & $\begin{array}{r}83 \\
72 \\
95 \\
100 \\
74 \\
75 \\
99 \\
85 \\
105 \\
103 \\
104 \\
101 \\
92 \\
80 \\
105 \\
90 \\
82 \\
75 \\
88 \\
100 \\
93\end{array}$ & $\begin{array}{l}135 \\
140 \\
140 \\
130 \\
135 \\
130 \\
135 \\
130 \\
139 \\
140 \\
148 \\
135 \\
145 \\
132 \\
150 \\
135 \\
145 \\
131 \\
133 \\
137 \\
138\end{array}$ & $\begin{array}{r}102 \\
92 \\
116 \\
113 \\
92 \\
97 \\
116 \\
105 \\
122 \\
125 \\
122 \\
125 \\
116 \\
94 \\
118 \\
118 \\
100 \\
98 \\
100 \\
116 \\
109\end{array}$ & $\begin{array}{c}118 \\
-\overline{-} \\
\overline{110} \\
110 \\
130 \\
- \\
127 \\
\overline{135} \\
123 \\
130 \\
114 \\
134 \\
128 \\
132 \\
122 \\
121 \\
130 \\
131\end{array}$ & $\begin{array}{c}125 \\
- \\
- \\
- \\
105 \\
110 \\
130 \\
- \\
123 \\
- \\
135 \\
123 \\
130 \\
118 \\
140 \\
122 \\
130 \\
122 \\
122 \\
127 \\
131\end{array}$ & $\begin{array}{l}117 \\
118 \\
105 \\
105 \\
116 \\
114 \\
119 \\
117 \\
124 \\
129 \\
120 \\
124 \\
120 \\
112 \\
121 \\
114 \\
117 \\
112 \\
110 \\
118 \\
118\end{array}$ & $\begin{array}{l}110 \\
112 \\
107 \\
105 \\
122 \\
116 \\
119 \\
115 \\
120 \\
118 \\
122 \\
126 \\
116 \\
114 \\
125 \\
114 \\
117 \\
112 \\
110 \\
118 \\
118\end{array}$ & $\begin{array}{l}113,5 \\
115 \\
106 \\
105 \\
119 \\
115 \\
119 \\
116 \\
122 \\
120 \\
121 \\
125 \\
118 \\
113 \\
123 \\
114 \\
117 \\
112 \\
110 \\
118 \\
118\end{array}$ & $\begin{array}{l}21,5 \\
25 \\
34 \\
25 \\
16 \\
15 \\
16 \\
14 \\
17 \\
20 \\
27 \\
10 \\
27 \\
19 \\
17 \\
21 \\
18 \\
19 \\
23 \\
19 \\
20\end{array}$ \\
\hline & & & & & & \multicolumn{2}{|c|}{ Summe: } & 2385,5 & 443,5 \\
\hline & & & & & & \multicolumn{2}{|c|}{ Durchschnitt: } & 113,6 & 21,11 \\
\hline
\end{tabular}

Sehlussfolgerung: Die absoluten Maasse der Schrägaulsteigenden Löhlein's weisen die Grenzen 10,5 und $12,6 \mathrm{~cm}$ auf, d. h. sie haben einen Spielraum von $2 \mathrm{~cm}$; der Durchsehnitt beträgt 11,36 $\mathrm{cm}$ und kommt damit dem Durchschnitt bei den allgemeinverengten Becken erheblich nahe (11,23). Ganz anders fällt die zu addirende Differenz aus. Sie zeigt fast durchweg hohe Zahlen, dem entspricht auch die Durchschnittsdifferenz von $21,11 \mathrm{~mm}$, die die normale um mehr als $0,5 \mathrm{~cm}$ übertrifft.

Tabelle $\mathrm{V}$ siehe folgende Seite.

Schlussfolgerung: Die Maasse bei diesen Becken weisen keinen einheitlichen Charakter auf wie man vieileicht hätte erwarten können. Im eirzelnen entsprechen die Maasse entweder mehr dem platten oder dem allgemeinverengten Becken. Die absoluten Maasse Löhlein's bewegen sich zwischen 9,6 und 
Tabelle V. Allgemeinverengte platte Becken.

\begin{tabular}{|c|c|c|c|c|c|c|c|c|c|}
\hline No. & C. $v$, & Tr. & C. d. & o.d. & o.s. & L. R. & L. L. & $\begin{array}{l}\text { Durch- } \\
\text { schnitt }\end{array}$ & Differ. \\
\hline 42 & 89 & $1 \supseteq 0$ & 104 & - & -- & 104 & 105 & 104,5 & 21,5 \\
\hline 43 & 90 & 122 & 111 & -- & - & 107 & 110 & 108,5 & 13,5 \\
\hline 44 & 90 & 120 & 100 & - & - & 96 & 102 & 99 & 21 \\
\hline 45 & 74 & 124 & 88 & 107 & 102 & 102 & 105 & $\mathrm{~J} 03,5$ & 20,5 \\
\hline 46 & 96 & 132 & 113 & 121 & 124 & 120 & 118 & 119 & 13 \\
\hline 47 & 93 & 131 & 114 & 120 & 117 & 114 & 118 & 116 & 15 \\
\hline 48 & 77 & 129 & 93 & 1.13 & 113 & 106 & 106 & 106 & 23 \\
\hline 49 & 89 & 117 & 114 & 113 & 113 & 108 & 113 & 110,5 & 6,5 \\
\hline 50 & 88 & 128 & 97 & 120 & 120 & 112 & 112 & 112 & 16 \\
\hline 51 & 90 & 122 & 106 & 110 & 110 & 106 & 106 & 106 & 16 \\
\hline 52 & 90 & 127 & 112 & 120 & 115 & 110 & 112 & 111 & 16 \\
\hline 53 & 95 & 126 & 113 & 121 & 121 & 115 & 117 & 116 & 10 \\
\hline 54 & 96 & 125 & 116 & 120 & 120 & 118 & 119 & 118,5 & 6,5 \\
\hline & & & & & & \multicolumn{2}{|c|}{ Summe: } & 1430.5 & 198,5 \\
\hline & & & & & & \multicolumn{2}{|c|}{ Durchschnitt: } & 110 & 15,27 \\
\hline
\end{tabular}

$12 \mathrm{~cm}$, der Durchschnitt ist $11 \mathrm{~cm}$; die Differenzen schwanken in relativ weiten Grenzen 6,5 und $21,5 \mathrm{~mm}$, entsprechend der Combination von allgemeinverengt und platt; die Durchschnittsdifferenz hält demgemäss die Mitte ond beträgt $15,3 \mathrm{~mm}$, sie kommt also damit der Normaldifferenz sehr nahe.

Tabelle VI. Schrägverschobene platte Becken.

\begin{tabular}{|c|c|c|c|c|c|c|c|c|c|}
\hline No. & C. v. & Tr. & C. d. & o. d. & 0. s. & L. R. & L. L. & $\begin{array}{l}\text { Durch- } \\
\text { schnitt }\end{array}$ & Differ. \\
\hline $\begin{array}{l}55 \\
56 \\
57 \\
58 \\
59 \\
60 \\
61 \\
62 \\
63 \\
64\end{array}$ & $\begin{array}{r}90 \\
58 \\
93 \\
105 \\
72 \\
87 \\
97 \\
105 \\
107 \\
100\end{array}$ & $\begin{array}{l}140 \\
140 \\
141 \\
133 \\
135 \\
137 \\
145 \\
143 \\
145 \\
140\end{array}$ & $\begin{array}{r}117 \\
77 \\
109 \\
122 \\
81 \\
101 \\
114 \\
127 \\
120 \\
118\end{array}$ & $\begin{array}{l}132 \\
123 \\
135 \\
118 \\
109 \\
127 \\
135 \\
128 \\
135 \\
135\end{array}$ & $\begin{array}{l}125 \\
117 \\
120 \\
112 \\
116 \\
118 \\
127 \\
123 \\
128 \\
128\end{array}$ & $\begin{array}{l}117 \\
114 \\
113 \\
119 \\
105 \\
119 \\
123 \\
120 \\
120 \\
125\end{array}$ & $\begin{array}{l}117 \\
110 \\
113 \\
119 \\
107 \\
115 \\
122 \\
120 \\
117 \\
123\end{array}$ & $\begin{array}{l}117 \\
112 \\
113 \\
119 \\
106 \\
119 \\
123 \\
120 \\
118,5 \\
124\end{array}$ & $\begin{array}{l}15 \\
28 \\
28 \\
14 \\
29 \\
18 \\
22 \\
23 \\
26.5 \\
16\end{array}$ \\
\hline & & & & & & \multicolumn{2}{|c|}{ 'Summe: } & 1171,5 & 219,5 \\
\hline & & & & & & \multicolumn{2}{|c|}{ Durchschnitt: } & 117,1 & 21,9 \\
\hline
\end{tabular}

Sehlussfolgerung: Die Maasse werden im wesentlichen bestimmt durch die Eigenschaft „platt“. Dementsprechend sind die Differenzen ziemlich hoch; im Durchschnitte $21,9 \mathrm{~mm}$, also fast gleich den einfach platten $(21,1 \mathrm{~mm})$. Die absoluten Maasse Löhlein's bewegen sich $z$ wischen 10,5 und 12,5 , und betragen im 
442 Steinbrecher, Die Schätzung der Transversa des Beckeneingangs.

Durchschnitt 11,7 (11,3 bei den platten). Auf die Asymmetrie und die daraus sich ergebenden Folgerungen soll weiter unten noch zurückgekommen werden.

Tabelle VII. Schrägverschobene bezw. schrägverengte Becken.

\begin{tabular}{|c|c|c|c|c|c|c|c|c|c|}
\hline No. & C. v. & Tr. & C. d. & o. d. & o. s. & L. R. & L. L. & $\begin{array}{l}\text { Durch- } \\
\text { schnitt }\end{array}$ & Differ. \\
\hline $\begin{array}{l}65 \\
66 \\
67\end{array}$ & $\begin{array}{l}128 \\
113 \\
131\end{array}$ & $\begin{array}{l}125 \\
125 \\
146\end{array}$ & $\begin{array}{l}147 \\
117 \\
129\end{array}$ & $\begin{array}{l}145 \\
100 \\
134\end{array}$ & $\begin{array}{l}125 \\
130 \\
124\end{array}$ & $\begin{array}{l}133 \\
110 \\
128\end{array}$ & $\begin{array}{l}120 \\
126 \\
120\end{array}$ & $\begin{array}{l}126,5 \\
118 \\
124\end{array}$ & $\begin{array}{c}-1,5 \\
7 \\
22\end{array}$ \\
\hline & & & & & & \multicolumn{2}{|c|}{ Summe: } & 368,5 & 28,5 \\
\hline & & & & & & \multicolumn{2}{|c|}{ Darchschnitt: } & 122,8 & 9,5 \\
\hline
\end{tabular}

Schlussfolgerung: Während die absoluten Maasse sich in viel geringeren Grenzen (11 und $13,3 \mathrm{~cm}$ ) halten, als man hätte erwarten können, zeigt uns ein Blick auf die Differenzen, dass hier von einer Regelmässigkeit schlechterdings nicht gesprochen werden kann. Im Becken No. 67 ist die Differenz ziemlich hoch (22) und in No. 65 wird sie sogar negativ! Es dürfte sich daraus ergeben, dass man bei hochgradig asymmetrischen Becken aus den Löhlein'schen Maassen kaum einen Schluss auf die Transversa machen kann.

Wir kommen nun zu der Frage, welchen Schluss auf die Asymmetrie eines Beckens erlauben die Schrägaufsteigenden? Diese Frage kann bei höheren Graden von Asymmetrie enorm wichtig werden (vergl. Fig. la, b, c). In den Becken No. 65, 66, 67 haben wir eine Verschiedenheit der Obliqui (des schrägen Durchmessers des Beckeneingangs) von 20, 30 und $10 \mathrm{~mm}$, diesen entsprechen Lnterschiede zwischen den schrägaufsteigenden Maassen rechts und links von 13,16 und $8 \mathrm{~mm}$, d. h. die Asymmetrie ist erheblich abgeschwächt, doch ist sie zu' diagnosticiren und als eine Asymmetrie im gleichen Sinne aufzufassen, d. h. dem grösseren Löhlein'schen Maasse entspricht der grössere Obliquus des Beckeneingangs. Dieser Satz gilt jedoch nur für die Unterschiede der Löhlein-Maasse, die mehr als $0,5 \mathrm{~cm}$ betragen. Unterschiede der Schrägaufsteigenden bis $0,5 \mathrm{~cm}$ lassen keinen sicheren Schluss auf die Asymmetrie eines Beckens zu.

Wir haben nämlich eine Reihe von Becken, die eine Verschiedenheit der Obliqui aufweisen, ohne dass in den Schrägaufsteigenden sich diese Asymmetrie deutlich erkennbar machte. Man braucht, um dies eklatant zu beweisen, nur das Becken No. 57 zu 
betrachten, das einen Unterschied der Obliqui von $1,5 \mathrm{~cm}$ zeigt, während die Schrägaufsteigenden als gleich gemessen werden mussten!

Das Ergebniss ist also kurz Folgendes: Haben wir LöhleinUnterschiede von mehr als $0,5 \mathrm{~cm}$, so liegt ein asymmetrisches Becken vor; der Grad der Asymmetrie ist nicht sicher zu erkennen. Die Asymmetrie der Obliqui verläuft in demselben Sinne, wie die der Schräganfsteigenden. Umgekehrt: Es kann eine stärkere Asymmetrie des Beckens vorliegen, ohne dass sie in den Schrägaufsteigenden zum Ausdruck kommt.

Figur 1.

a)

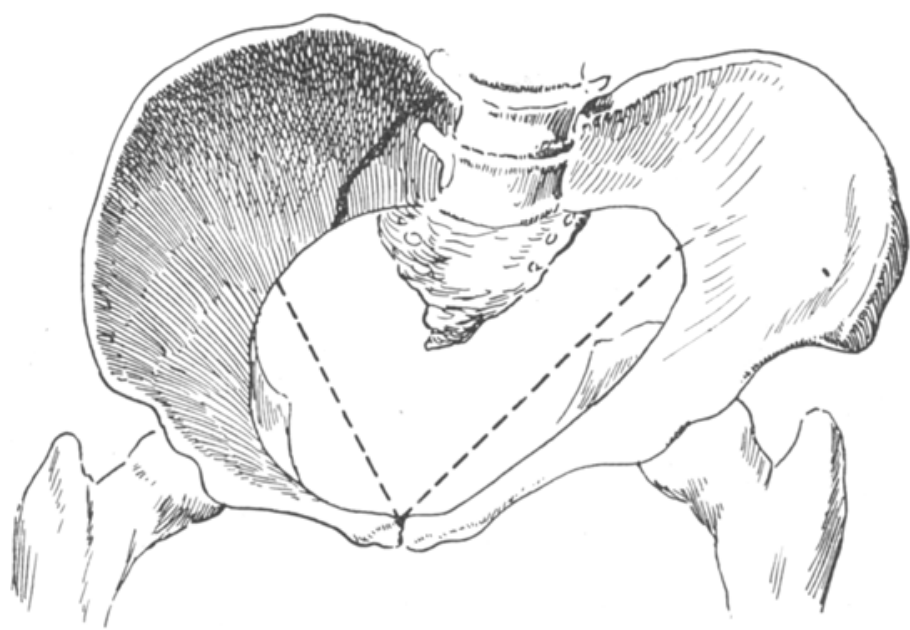

a) Darstellung eines Nägele'schen Beckens der Giessener Frauenklik in der Aufsicht. Die punktirten Linien entsprechen den schräg aufsteigenden Durehmessern: Der grössere schräge Durchmesser links $=126 \mathrm{~mm}$ entspricht der Verengerung, der kleinere rechts $=110 \mathrm{~mm}$ der normalen Beckenseite.

Tabelle VIII. Querverengte Becken.

\begin{tabular}{|c|c|c|c|c|c|c|c|c|c|}
\hline No. & C. v. & Tr. & C. d. & $0 . d$. & o. s. & L. P. & L. I. & $\begin{array}{l}\text { Dureh- } \\
\text { schnitt }\end{array}$ & Differ. \\
\hline $\begin{array}{l}68 \\
69 \\
70 \\
71 \\
72 \\
73 \\
74\end{array}$ & $\begin{array}{l}112 \\
120 \\
112 \\
100 \\
113 \\
109 \\
125\end{array}$ & $\begin{array}{l}120 \\
130 \\
127 \\
120 \\
110 \\
123 \\
127\end{array}$ & $\begin{array}{l}- \\
\overline{132} \\
110 \\
132 \\
128 \\
140\end{array}$ & $\begin{array}{l}- \\
- \\
- \\
- \\
- \\
123\end{array}$ & $\begin{array}{l}- \\
- \\
- \\
- \\
- \\
127\end{array}$ & $\begin{array}{l}113 \\
124 \\
118 \\
116 \\
116 \\
124 \\
121\end{array}$ & $\begin{array}{l}115 \\
120 \\
115 \\
116 \\
114 \\
116 \\
125\end{array}$ & $\begin{array}{l}114 \\
122 \\
118 \\
116 \\
115 \\
120 \\
123\end{array}$ & $\begin{array}{r}6 \\
8 \\
9 \\
4 \\
-5 \\
3 \\
4\end{array}$ \\
\hline & & & & & & \multicolumn{2}{|c|}{ Summe: } & 828 & 99 \\
\hline & & & & & & \multicolumn{2}{|c|}{ Durchsehnitt: } & 118,3 & 4,1 \\
\hline
\end{tabular}


444 Steinbrecher, Die Schätzung der Transversa des Beckeneingangs.

Schlussfolgerung: Die absoluten Maasse Löhlein's bewegen sich in den Grenzen von 11,3 und 12,5 cm; ihr Durchschnitt ist $11,8 \mathrm{~cm}$. Die zu addirenden Differenzen sind, wie zu erwarten war, sehr niedrig, sie schwanken zwischen - 5 und $+9 \mathrm{~mm}$; sie zeigen also im Ganzen einen erfreulich geringen Spielraum; der Durchschnitt ist 0,4 em. Gelingt es uns demnach aus den sonstigen Beckenmaassen auf ein querverengtes Becken zu schliessen - auch die Trochanterenentfernung dürfte in diesem Falle nicht ausser Acht gelassen werden - so wäre unseres Erachtens ein Schluss aus den Schrägaufsteigenden auf die Transversa erlaubt.

Figur 1.

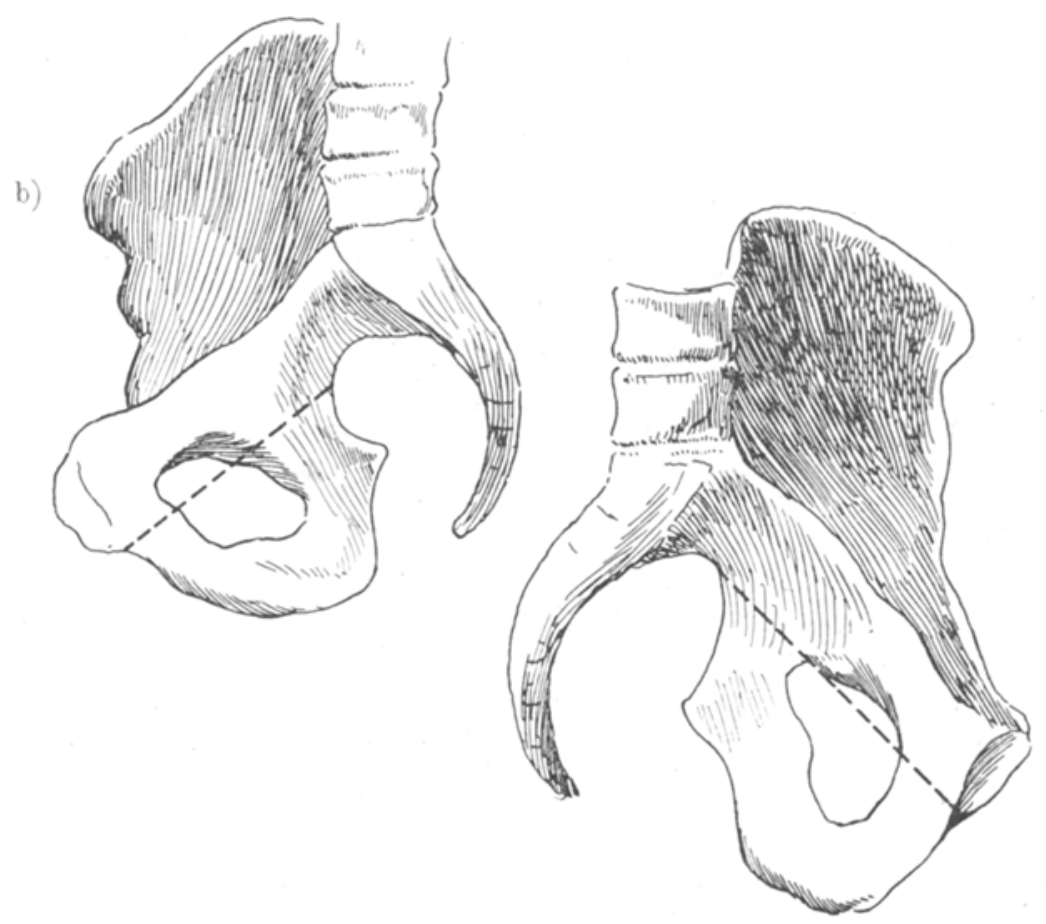

b) Gesunde rechte Hälfte des obigen Beckens.

c) Verschobene linke Hälfte des obigen Beckens. Die Verschiedenheit des schrägaufsteigenden Durchmesser beruht auf der Abflachung und Streckung des linken Hüftbeins. Stellen wir uns beide Hüftbeine als Theile eines ungleichen Beckengürtels vor, dessen beide Seiten rerschiedene Bogenspannung haben, so muss dem stärker gespannten Bogen der kleinere Radius und die kleinere Sehne entsprechen, -...- so hier der stärlker gespanten rechten Beckenseite der kleinere schräge Durchmesser. 
Wenn wir nun zum Schlusse das Facit unserer Untersuchungen ziehen wollen, so kommen wir, wie wir uns nicht verhehlen dürfen, zu einem Resultate, das von dem Löhlein'schen in mancher Beziehung abweicht. Wir haben gesehen, dass es nicht angängig ist, eine Normal- oder Gesammtdifferenz aufzustellen (nach Löhlein 2,06 cm), die für einige Beckenformen passend wäre. Diese Differenz ist im Allgemeinen keine Constante; doch kann die bei den einzelnen Beckenformen gefundene Differenz für diese einzelnen Beckenformen als Constante angesehen werden, und dies ist das positive Ergebniss der Arbeit.

Gelingt es uns aus den übrigen Maassen einen Schluss auf die Beckenform zu machen, so sind wir bis zu einem gewissen Grade der Wahrscheinlichkeit in der Lage, aus den Schrägaufsteigenden Löhlein's einen Schluss auf die Transversa des Beckeneingangs zu machen; wir haben demgemäss zu der halben Summe der Schrägaufsteigenden die jeweilige Durchschnittsdifferenz zu addiren, also bei einem platten Becken $21 \mathrm{~mm}$, bei einem allgemein verengten $13,5 \mathrm{~mm} \mathrm{u.} \mathrm{s.} \mathrm{w.}$

Die Anwendung der Löhlein'schen Methode kann in einigen Fällen versagen oder uns manchmal nicht die diagnostische Sicherheit geben, die wir für unsere therapeutischen Maassnahmen brauchen. Wenn wir in solchen Fällen Umschau halten nach einer andern Methode, die das nach Löhlein gefundene Resultat controlliren soll, so kommen nach unserer Meinung nur zwei Methoden in Betracht. - beide sind instrumentelle - nämlich die von Skutsch und die 3. Methode Küstner's. Das Instrument von Skutsch liefert gute Resultate, ist aber an sich und in seiner Anwendung zu complicirt und nach unserem Dafürhalten eher in einer geburtshülflichen Klinik verwendbar als in der Praxis. Demgegenüber ist die Methode Kü'stner's die denkbar einfachste. Küstner bezweckt die directe Messung der Transversa mit einem scheerenförmigen Instrument, dessen Branchen in je eine Halbkugel von $2 \mathrm{~cm}$ Radius auslaufen. Wird das Instrument eingeführt, so ist die Scheere geschlossen, und die beiden Halbkugeln ergänzen sich zu einer ganzen Kugel. Um die Messung auszuführen, öffnet man die Scheere, so weit es geht, und controllirt die beiden Messpunkte mit der eingeführten Hand. Die gemessene Entfernung kann an einer aussen an den Griffen befindlichen Scala abgelesen werden. Das Instrument, das an sich einfach und praktisch ist, hat den Nachtheil, dass es nur in den Fällen anwendbar ist, wo 
die Weichtheile der Geburtswege einigermaassen debnbar und aufgelockert sind. Ferner ist beachtenswerth, dass man die Wichtigkeit der Scala vorher genau controllirt. In der vorliegenden Arbeit wurden bei sämmtlichen Becken die Tranversa auch mit dem Küstner: schen Instrument gemessen. Die Messung ergab im Allgemeinen gute Resultate. In der beigefügten Tabelle sind von 12 Becken, die ganz beliebig gewählt sind, die Resultate mitgetheilt. Da die Fehlerquellen gering sind $(2-3 \mathrm{~mm}$ im Durchschnitt) und in demselben Sinne ausgefallen sind, war die Mittheilung aller Resultate überflüssig.

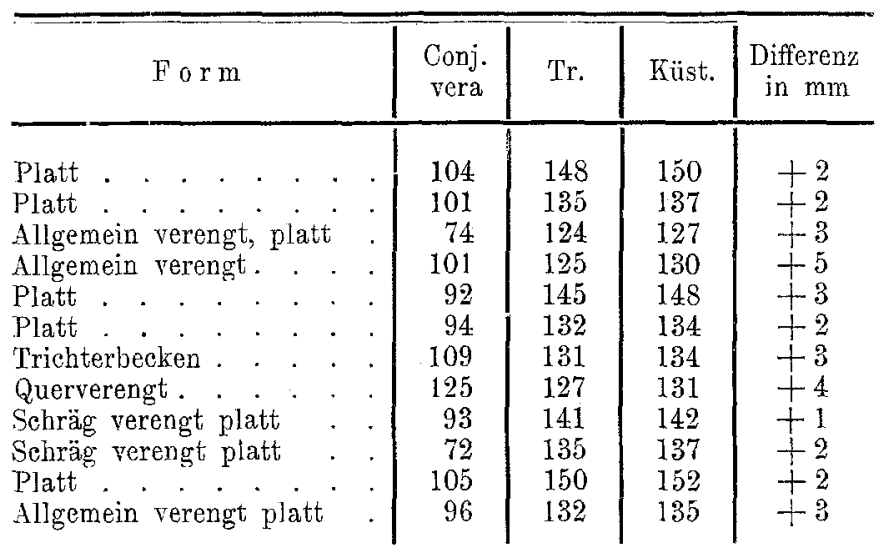

Wje schon gesagt, beträgt die Durchschnittsdifferenz 2-3 mm und zwar ist das Küstner'sche Maass das grössere. Es kamen auch Differenzen von 5, ja von $7 \mathrm{~mm}$ vor, doch nur selten. Vielleicht ist ein Theil der Fehlerquellen noch dem Instrument zur Jast zu legen. Jedenfalls ist es rathsam, vorher die durchschnittliche Fehlerquelle an Bänderbecken auszuprobiren, dann wird man in den Fällen, wo man das Instrument anwenden kann, ein befriedigendes Resultat erhalten.

Klinische Werthung der Löhlein'schen Methode. Aus den vorliegenden Messungen ergiebt sich, dass man durch das von I,öhlein angegebene Verfahren sehr wohl im Stande ist, durch Berücksichtigung der Beckenform die Transversa des Beckeneingangs mit einer gewissen Wahrscheinlichkeit zu berechnen. Die Methode ist auch an der Lebenden einfach und für die Untersuchten weniger schmerzhaft als die Vessung der Diagonalis. Für den Anfänger erscheint sie uns von besonderem Vortheile, weil sie 
den Lernenden zu einer genauen Austastung des Beckens zwingt und ihm eine leidliche Vorstellung von der grösseren oder kleineren Querspannung des Beckens giebt. Das Wichtige unserer Untersuchungen liegt. in dem Nachweis, dass die constante Differenz zwischen Transversa and Löhlein-Durchschnitt $\left(\frac{l_{1}+l_{2}}{2}\right)$ je nach der Beckenform von $4 \mathrm{~mm}$ bis zu $21 \mathrm{~mm}$ variirt. Wir dürfen also nicht immer denselben Werth für die Constante einsetzen, sondern werden bei platten Becken ca. $20 \mathrm{~mm}$, bei allgemeinverengten $13,5 \mathrm{~mm}$, bei allgemeinverengt-platten Becken $15 \mathrm{~mm}$ zum Löhlein-Durchschnitt $\frac{l_{1}+l_{2}}{2}$ addiren müssen, um das richtige Maass der Transversa zu finden. Je grösser die Querspannung und die Abflachung des Beckens, um so grösser wird die constante Differenz, je kleiner die Querspannung, um so geringer wird die Differenz (vergl. die folgenden von Kroemer entworfenen Schemata).

Figur 2. $(a-f$.

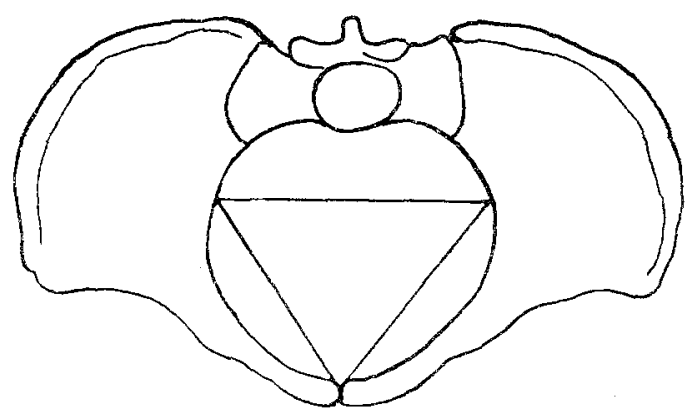

a) Normales Becken. Tr. (Küstner) $=\mathrm{L}+1,5$.

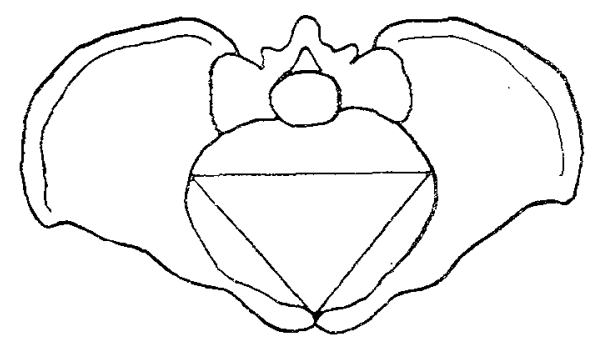

b) Allgemein verengtes Becken. Tr. $=\mathrm{L}+1,35$. 
448 Steinbrecher, Die Schätzung der Transversa des Beckeneingangs.

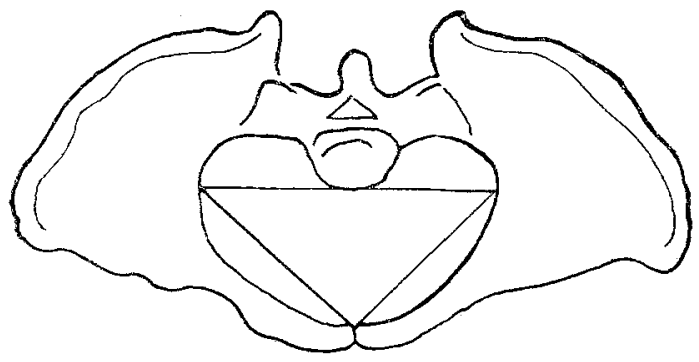

c) Plattes Becken. Tr. $=$ L. $+2,1$.

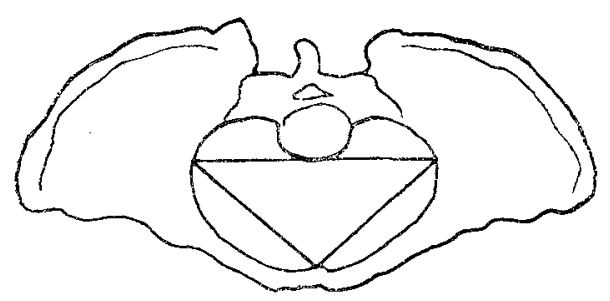

d) Allgemein plattes Becken. $\mathrm{Tr} .=\mathrm{L}+1,5$.

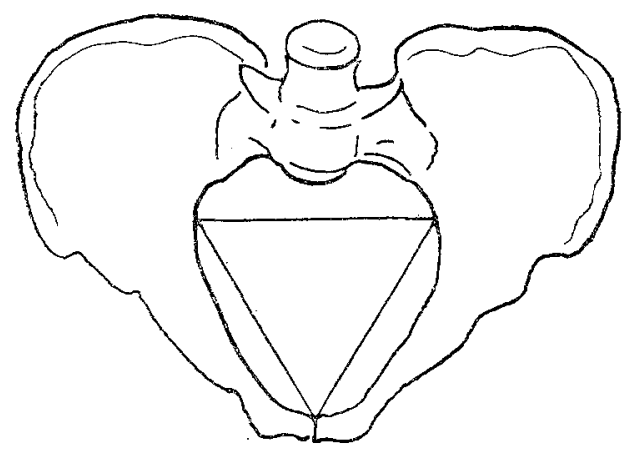

e) Quer verengtes Becken. Tr. $=\mathrm{L}+0,4$.

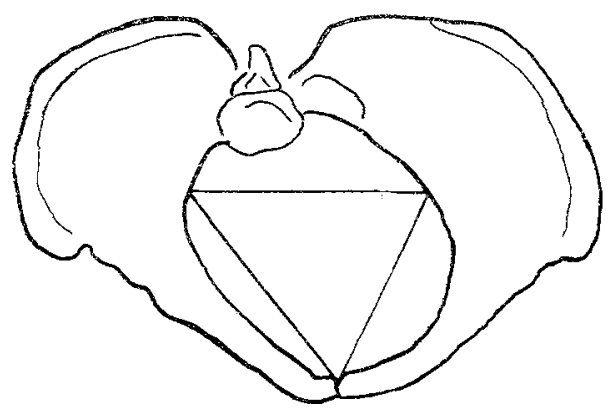

f) Schräg verengtes Becken. Tr. nicht in Vergleich zu setzen. $L \geq I$. 
Wenn man in der Beckenaufsicht die Endpunkte der beiden Schrägaufsteigenden mit einander verbindet, so erhält man ein Dreieck, dessen Basis, d. i. die Verbindungslinie, ziemlich genau die Projection der Transversa des Beckeneingangs darstellt. Für die Praxis kann man die Verbindungslinie gleich der Transversa setzen. Die so construirte Dreiecksfigur wechselt wach der Art der Beckenform. Bei unregelmässig verengten z. B. schrägen Becken werden wir ein ungleichseitiges Dreieck erhalten und aus der Ungleichheit der Seiten schliessen können, welche Beckenhälfte als verengt bezw. als verschoben anzusehen ist (Vergleich das Beispiel Fig. 1). Bei regelmässig verengten Becken, sowie bei den normalen Formen werden wir ein gleichschenkeliges Dreieck erhalten; das Letztere wird bei den infantilen, querverengten Beckenformen nahezu ein gleichseitiges Dreieck werden. Je niedriger das gleichschenkelige Dreieck erscheint, um so mehr werden wir auf eine Abplattung und gesteigerte Querspannung schliessen können. Am Bänderbecken sind diese Dreiecke natürlich mit Leichtigkeit. zu construiren. An der Lebenden werden wir von Fall zu Fall gezwungen sein, mit Hülfe der in dieser Arbeit aufgestellten Zahlenrelationen die Dreiecksbasis-Transversa zu berechnen, d. h. wir müssen unter Berücksichtigung aller äusseren und inneren Maasse die allgemeine Beckenform erkannt haben, ehe wir die Construction entwerfen können. Der Geübte wird mit Wahrscheinlichkeit die Beckenform richtig schätzen. Wir sind uns aber wohl bewusst, dass uns bei der Beckenschätzung zuweilen grosse Schwierigkeiten entgegentreten und sind uns in Folge dessen über die Grenzen der Leistungsfähigkeit des Verfahrens im Klaren. Auch Löhlein betonte ja schon, dass seine Berechnung nur ein Theil im Gesammtbilde der Beckenschätzung sein könne. Wenn wir auf die praktischen Verhältnisse zurückgreifen, so werden wir Fehlerquellen, die selbst bis zu $5 \mathrm{~mm}$ sich erstrecken, gern in den Kauf nehmen, wenn andererseits wir durch unser Verfahren in die Lage versetzt werden, etwas mehr über ein Becken zu erfahren, als es die bisherige Conj. diag.- und vera-Bestimmung ermöglicht. Kroemer macht auch mit Recht auf folgende Relation aufmerksam: Aus unsern vergleichenden Messungen geht hervor, dass die Bestimmung der Transversa durch den Küstner'schen Beckenmesser im Allgemeinen zuverlässige Maasse ergiebt (vergl. die Schlusstabelle). Für die Praxis können wir das Küstner'sche Maass ==Transversa setzen. In allen denjenigen Fällen, in welchen man an der Leben- 
450 Steinbrecher, Die Schätzung der Transversa des Beckeneingangs.

den die Methode Küstner III anwenden kann, will Kroemer die Beckenform durch den Vergleich der Löhlein-Messung mit dem $\mathrm{K}$ üstner-Resultat bestimmen und zwar in folgender Weise: Setzen wir das mit dem Küstner-Zirkel gewonnene Maass=Transversa des Beckeneingangs $=\mathrm{Tr}$, so ist $\operatorname{Tr}=\frac{l_{1}+l_{2}}{2}+\mathrm{C} \quad(\mathrm{C}=$ Constante $)$. In dieser Gleichung haben wir $\operatorname{Tr}$ und $\frac{l_{1}+l_{2}}{2}$ durch Messung bestimmt; daraus ist die Constante $\mathrm{C}=\operatorname{Tr}-\frac{\mathrm{l}_{1}+\mathrm{l}_{2}}{2}$ leicht zu berechnen, und die nun mögliche Construction der Beckenform mit dem oben erwähnten Dreieek gewährt uns ein Bild über die Form des Beckeneingangs (vergl. die schemat. Darstellungen in Fig. II bis VII). Ist z. B. in obiger Gleichung $\mathrm{C}=4 \mathrm{~mm}$, so liegt ein querverengtes Becken vor, ist die Constante $\mathrm{C}=20 \mathrm{~mm}$ und darüber, so haben wir ein plattes Becken vor uns. Liegt die Constante zwischen 10 und $15 \mathrm{~mm}$, so ist mit einiger Wahrscheinlichkeit auf ein allgemein verengtes Becken zu schliessen.

Es wird unsere Aufgabe in Zukunft sein, unsere Resultate an der Lebenden und am Leichenmaterial nachzuprüfen, hoffentlich werden meine bescheidenen Resultate einer Nachprüfung gewürdigt.

\section{Lit e rat us.}

Ahlfeld, Lehrbuch der Geburtshülfe. 1903. III. Aufl. Bumm, Grundriss der Geburtshülfe. 1903.

Fritsch, Geburtshülfe. 1904.

Kaltenbach, Juehrbuch der Geburtshülfe. 1893.

Küstner, Verhandlungen auf dem I. Congress für Gynälologie, München. 1886.

Löhlein, Zeitschr. f. Gynäk. und Geburtshülfe. Bd. XI.

Olshausen und Veit, Lehrbuch der Geburtshülfe. 13. Aufl.

Schauta, Operative Geburtshülfe. II. Aufl. 1892.

Skutsch, Beckenmessung an der lebenden Frau. Jena. 1887.

Spiegelberg, Lehrbuch der Geburtshülfe. 2. Aufl. 1882.

v. Winckel, Handbuch der Geburtshülfe. II. Band.

Zweifel, Lehrbuch der Geburtshülfe. 5. Aufl. 1903. 